\title{
Post -Operative Bilateral Renal Sub capsular Hematoma, Successfully Treated with Laparoscopic Drainage - A Case Presentation
}

\author{
Sameer Bhuwania ${ }^{1^{*}}$, Neeraj Nirala ${ }^{2}$, Rajesh Goel ${ }^{3}$ and Sanjiv Saxena ${ }^{4}$ \\ ${ }^{1}$ MBBS, MD (Medicine), Pushpawati Singhania Research Institute, New Delhi, India. \\ ${ }^{2}$ MBBS, MD (Medicine), DNB (Nephrology), Pushpawati Singhania Research Institute, New Delhi, India. \\ ${ }^{3}$ DNB (Medicine), Consultant Nephrology, Pushpawati Singhania Research Institute, New Delhi, India. \\ ${ }^{4}$ MBBS, MD(Medicine), DNB(Nephrology), Head of Department and Chairman, Dept. of Nephrology, Pushpawati Singhania Research Institute, \\ New Delhi, India.
}

*Corresponding Author: Sameer Bhuwania, MBBS, MD (Medicine), Pushpawati Singhania Research Institute, New Delhi, India.

Received date: February 25, 2021; Accepted date: March 25, 2021; Published date: April 05,2021

Citation: Bhuwania S., Nirala N., Goel R. and Saxena S. (2021) Post -Operative Bilateral Renal Sub capsular Hematoma, Successfully Treated with Laparoscopic Drainage - A Case Presentation. J. Surgical Case Reports and Images 4(2); DOI:10.31579/2690-1897/063

Copyright: (C) 2021 Sameer Bhuwania, This is an open access article distributed under the Creative Commons Attribution License, which permits unrestricted use, distribution, and reproduction in any medium, provided the original work is properly cited.

\begin{abstract}
Page kidney is a rare phenomenon which present as uncontrolled hypertension and hypertensive emergencies. Here we bring to notice a case presenting with anuria and rapid progressive renal failure. We have also tried to focus on the use of imaging for diagnosis and benefits of prompt and effective therapy causing complete recovery of the kidneys.
\end{abstract}

Keywords: page kidney, anuria, rare DJ stent complications

\section{Introduction}

phenomenon was first described by Irvin Page in 1939 in animal experiments resulting from external compression of the kidney. It is a rare, treatable cause of secondary hypertension mediated by activation of the renin-angiotensin-aldosterone system (RAAS) [1]. Causes include bleeding around the kidney from blunt trauma, sports injury or iatrogenically following a procedure [1, 2]. Although not very uncommon, anuria is rarely a presentation in such a case since the other kidney takes over the function when one is injured. In our case, there was bilateral injury to the renal parenchyma causing such a presentation.

\section{Case presentation}

\begin{tabular}{|l|l|l|l|}
\hline & Baseline & $\begin{array}{l}\text { Pre-operative (At our } \\
\text { centre) }\end{array}$ & Post-operative Day 15 \\
\hline Hemoglobin $(\mathrm{g} \%)$ & 11.5 & 7.2 & 9.0 (Blood Transfused) \\
\hline Total Leukocyte count $\left(\right.$ per $\mathrm{mm}^{3)}$ & 4200 & 6500 & 4500 \\
\hline Urea (g\%) & 25 & 250 & 58 \\
\hline Creatinine (mg\%) & 0.7 & 6.8 & $3.2>0.8$ \\
\hline Potassium & 3.8 & 5.0 & 3.5 \\
\hline
\end{tabular}

Table 1 - showing the various blood investigations of the patient.

After complete evaluation, urological advice and high risk consent, laparoscopic capsulotomy and drainage of bilateral sub capsular hematoma under general anaesthesia was done. Over the next few hours, the patient started to pass urine at the rate of $200 \mathrm{ml} / \mathrm{hr}$. He was shifted to
A 57 year-old male non diabetic, non hypertensive with a normal baseline renal function, presented with bilateral renal calculi on routine workup. He underwent bilateral retrograde intra-renal surgery for stone removal for the same (elsewhere). Post operatively, though initially doing well, he developed anuria which could not be explained by the operating team. Suspecting a case of obstructive uropathy, surgeons performed bilateral DJ stenting of the kidneys but to no effect. On further worsening he was brought to our hospital for evaluation and treatment. At our centre, Clinical examination showed BP-180/110, mild pallor and pedal oedema. Laboratory investigations showed a creatinine levels upto $6.8 \mathrm{mg} \%$ and deranged blood parameters (Table 1). CT scan of abdomen was done which showed bilateral sub capsular hematoma (Picture 1).

ICU and kept under observation. In the coming days, patient condition improved, his uremic symptoms resolved and renal function became normal (Table 1). 


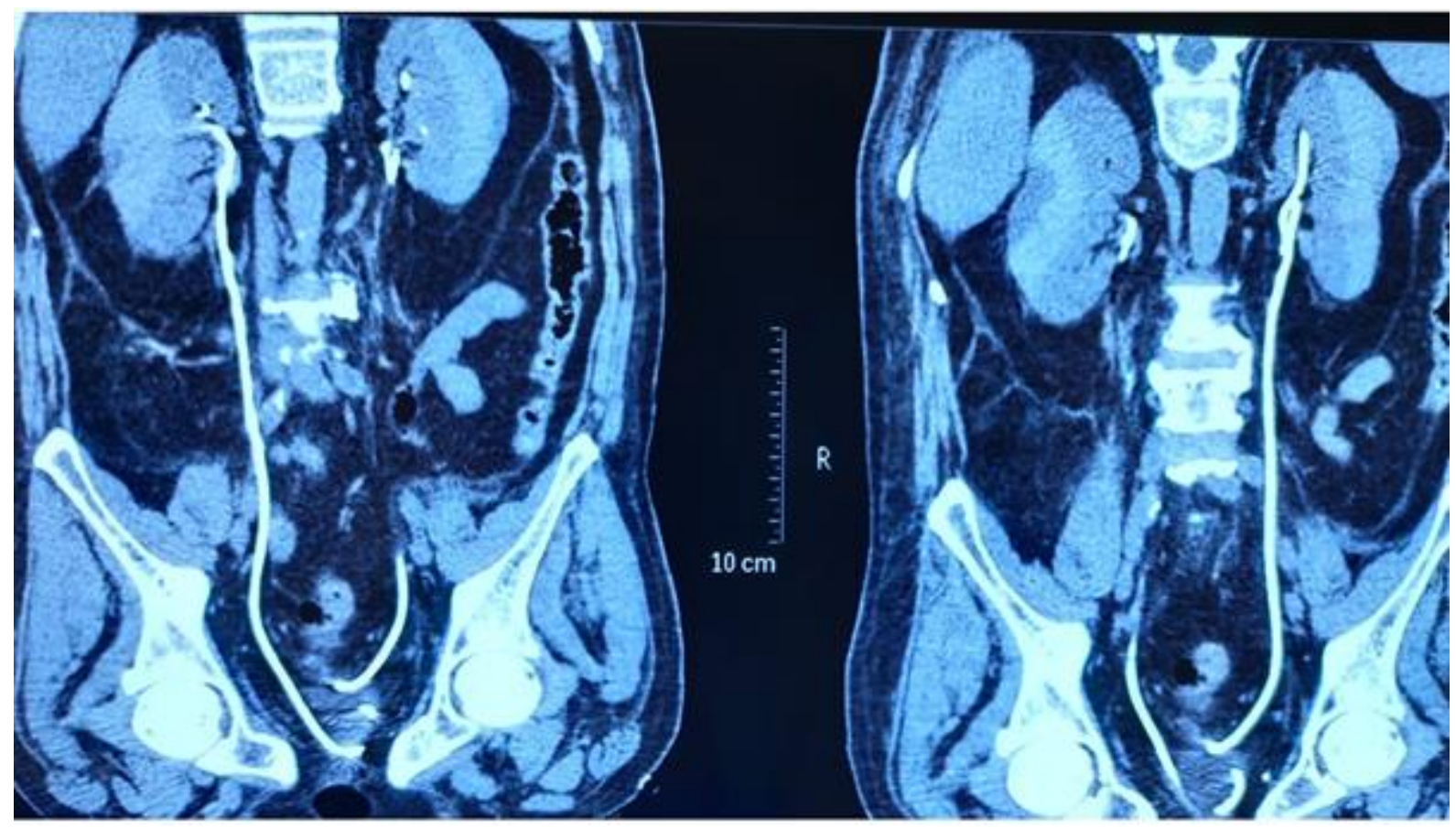

Figure 1. None contrast CT scan showing presence of bilateral sub capsular hematoma compressing the renal parenchyma with bilateral DJ stent in situ (Arrows).

\section{Discussion}

The kidneys are surrounded by a tough fibrous capsule with limited space. Even small amount of fluid under the capsule can cause compression of the kidney parenchyma resulting in Page kidney. Bleeding around the kidney may result from blunt trauma or iatrogenic (following biopsy) are few of the causes implicated [3]. Decreased perfusion of the intrarenal vessels due to external compression leads to the release of renin, activation of RAAS and hypertension. The resultant microvascular ischemia is similar to that shown in the Goldblatt experiment [3].

Page kidney is most often diagnosed based upon imaging studies in a case with relevant history and hypertension. Ultrasonography and computed tomography are the most frequently used modalities, with the latter being more informative [4]. Medical treatment of hypertension may is required for short period until a definitive procedure is undertaken. Interventions include open or percutaneous drainage of the compressing collection. If the collection has been of long standing, a fibrocollagenous shell may have formed, and this may require decapsulation [3]. Elevation of blood pressure is permanent in some cases, and lifelong antihypertensive drug therapy may be needed [5].

\section{Conclusion}

Through this case we have tried to bring to notice a rare complication of a very commonly procedure. Further we have stressed on the importance of comprehensive clinical approach and appropriate use of imaging when dealing with such a case.

\section{Conflict of interest}

No conflict of interest

\section{Funding}

None.

\section{References}

1. Haydar A, Bakri RS, Prime M, Goldsmith DJ. (2003) Page kidney--a review of the literature. J Nephrol. 16(3):329-33.

2. Dopson SJ, Jayakumar S, Velez JC. (2009) Page kidney as a rare cause of hypertension: case report and review of the literature. Am J Kidney Dis. 54(2):334-9.

3. Vaidya PN, Rathi BM, Finnigan NA. (2020) Page Kidney. Stat Pearls Publishing.

4. Arslan S. (2017) Bilateral nontraumatic recurrent Page kidney. Radiol Case Rep. 12(3).

5. Smyth A, Collins CS, Thorsteinsdottir B, Madsen BE, Oliveira GH, Kane G, Garovic VD. (2012) Page kidney: etiology, renal function outcomes and risk for future hypertension. J Clin Hypertens (Greenwich). 14(4):216-21. 